\title{
Role of long-range exact exchange in polaron charge transition levels: The case of MgO
}

\author{
Dahvyd Wing, ${ }^{1}$ Jack Strand, ${ }^{2}$ Thomas Durrant $\odot,{ }^{2}$ Alexander L. Shluger, ${ }^{2}$ and Leeor Kronik ${ }^{1}$ \\ ${ }^{1}$ Department of Materials and Interfaces, Weizmann Institute of Science, Rehovoth 76100, Israel \\ ${ }^{2}$ Department of Physics and Astronomy, University College London, Gower Street, London WC1E 6BT, United Kingdom
}

(Received 5 June 2020; accepted 29 July 2020; published 24 August 2020)

\begin{abstract}
Predicting the degree of localization and calculating the trapping energies of polarons in insulators by density functional theory (DFT) is challenging. Hybrid functionals are often reparametrized to obtain accurate results and the a priori selection of these parameters is still an open question. Here we test the accuracy of several range-separated hybrid functionals, all reparametrized to produce an accurate band gap, by calculating the charge transition levels (CTLs) of experimentally well-studied hole polaron defect centers in $\mathrm{MgO}$. We show that the functional with screened long-range exact exchange is moderately but consistently more accurate than functionals which do not include long-range exact exchange. We provide evidence that the source of the improved accuracy is the eigenvalue associated with the valence band maximum of the bulk material. We discuss the extent to which this accuracy relates to Koopmans' compliance of the defect energy level.
\end{abstract}

DOI: 10.1103/PhysRevMaterials.4.083808

\section{INTRODUCTION}

Point defects in insulators and semiconductors can have several charge states, owing to the trapping of extra electrons or holes. Their energies are characterized by charge transition levels (CTLs) [1,2], which are formally defined as the Fermi energy at which the formation energy of two different charge states of a defect center are equal. Density functional theory (DFT) is often used to assist in identifying and characterizing defects corresponding to experimentally measured CTLs, as well as to predict the CTLs of defects in a given material [3]. DFT typically provides reasonably reliable results for deep states in the band gap, but struggles with shallow states [4]. Challenging examples are the so-called polaron states, where electrons or holes are trapped by significant lattice polarization, either in a perfect crystal $[5,6]$ or at pre-existing defects $[7,8]$. Polarons play crucial roles in various material properties, e.g., conductivity [5,9], chemical reactivity [10], and optical absorption and emission [11,12]. In the context of polarons, the energy difference between the conduction (valence) band and the CTL of an electron (hole) polaron defect is the trapping energy of the polaron.

The difficulties of DFT in describing small polaron states, such as hole polarons which localize on a single oxygen ligand of a defect in metal oxides, are well documented [13-15]. Local or semilocal functionals typically fail qualitatively for small polarons by predicting, erroneously, that the charge carrier is delocalized over several lattice sites. Several clever schemes have been suggested to solve this problem $[16,17]$. Within standard DFT, hybrid functionals are a natural solution to this problem as they can properly localize polarons, though only if a large enough fraction of exact exchange is used [13-15]. This raises the question of how to choose an appropriate fraction of exact exchange in hybrid functionals to yield accurate CTLs for small polarons.
It has been suggested that the fraction of exact exchange in a hybrid functional should be fit to reproduce the bulk fundamental band gap of a material [18]. This would reduce the derivative discontinuity [19-21] of the functional for this material and thus the curvature of the functional with respect to a fractional number of electrons [22-24], which has been linked to the spurious delocalization of small polarons $[16,25]$. However, while this procedure produces fitted hybrid functionals that may localize small polarons, experience has shown that CTLs of defects calculated by such functionals are inaccurate [26]. This has been linked to the fact that CTL calculations depend on the position of the band edges, and the valence band maximum (VBM) and conduction band minimum (CBM) eigenvalues for different hybrid functionals, which are fit for the band gap, can shift by several tenths of an $\mathrm{eV}$ [27]. Thus the question can be rephrased as: Which of the hybrid functionals fit to the correct band gap would yield the best positions of the band edges and thus the most accurate CTLs for small polarons?

Recently it has been proposed that the fraction of exact exchange can be tuned to satisfy Koopmans' theorem for a localized defect level in a supercell [28-33]. Koopmans' theorem in DFT [19,34], also known as the ionization potential theorem, says that the highest occupied Kohn-Sham eigenvalue (in this case the occupied defect level) should be equal to the ionization potential calculated by total energy differences (i.e., by the $\Delta$-SCF method). It has been shown that this is synonymous with removing the curvature of the total energy with respect to a fractional number of electrons [23]. Specifically, the recent work of Deák et al. [28] fitted two parameters that control the amount of exact exchange in the Heyd-ScuseriaErnzerhof (HSE) hybrid functional [35] so as to reproduce the bulk fundamental band gap and satisfy Koopmans' theorem for a defect. HSE is a range-separated functional which formally has no long-range exact exchange, i.e., no exact 
exchange contribution arising from the long-ranged part of the Coulomb interaction. Their fitting procedure effectively led to setting the range-separation parameter to zero, such that it essentially became a global hybrid functional resembling the Perdew-Burke-Ernzerhof hybrid functional (PBE0) [36,37] with a somewhat modified fraction of exact exchange.

Long-range exact exchange is known to affect eigenvalues more than total energy differences [3,38-40]. This can be seen in Ref. [28] where satisfying Koopmans' theorem was in large part achieved by correcting the positions of the eigenvalues. This observation, together with experience which shows that long-range exact exchange is crucial to satisfying Koopmans' theorem for finite systems while still retaining the overall accuracy of the functional [41], leads us to hypothesize that using screened long-range exact exchange in a rangeseparated hybrid functional [42-45] may be a key ingredient to improving the position of VBM and CBM eigenvalues in CTL calculations and thus in the accurate prediction of the trapping energies of small polarons.

To test this hypothesis we use several range-separated hybrid functionals with different long-range behavior, each fit to obtain the fundamental band gap, to calculate the CTLs of hole polarons. Polarons self-trap in many materials, e.g., halides, oxides, cuprates, or manganates [46,47], but their CTLs are often controversial. Since we are seeking quantitative comparison, in this work we chose to focus on a prototypical dielectric material $\mathrm{MgO}$ and to investigate two well studied defect centers which form hole polarons: a substitution of a lithium atom on a magnesium site, called an [Li] center, and a magnesium vacancy, called a $\mathrm{V}$ center [48]. We investigate potential sources of deviations of obtained CTLs from experimental measurements, especially the position of the VBM eigenvalue and the satisfaction of Koopmans' theorem. We demonstrate that a functional with screened long-range exact exchange yields somewhat improved CTLs over functionals that do not have long-range exact exchange.

\section{METHODOLOGY}

\section{A. Selection of functionals}

In this study we use several range-separated hybrid functionals which address short- and long-range exchange differently, as summarized in Fig. 1. The first type is the HSE functional [35], which includes an amount of exact exchange in the short range that diminishes with increasing interelectron distance such that it has no long-range exact exchange. HSE, as well as reparametrized versions of it, have been used extensively in the literature to study defect properties $[3,18]$.

The second type is the PBE0 truncated long-range (PBE0TC-LRC) functional [49], which uses a constant fraction of exact exchange in the short range until a specified cut-off distance, at which it sets exact exchange to zero. It has been used in several defect studies and generally yielded results that successfully reproduced experimental findings [31,50,51]. In this study, PBE0-TC-LRC has the fraction of exact exchange set such that it has approximately the correct $\frac{1}{\epsilon_{\infty} r}$ behavior [52] until exact exchange is cutoff at an intermediate range.

The third type of functional is the screened range-separated hybrid (SRSH) $[42,44,45]$, which is similar to HSE in terms

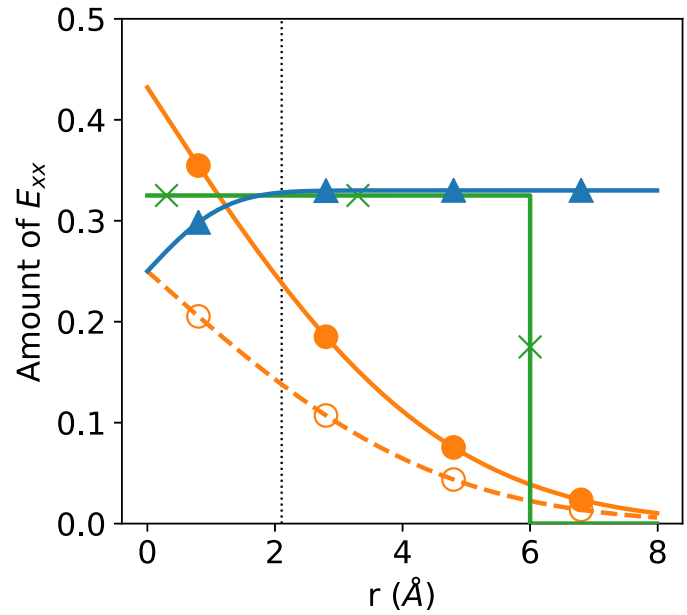

FIG. 1. Amount of exact exchange included in the three functionals investigated in this work as a function of the interelectron separation $r$. All three functionals are fit to reproduce the band gap of $\mathrm{MgO}$ : fitted HSE (orange filled circles), PBE0-TC-LRC (green x's), and SRSH (blue triangles). HSE in its original form (orange open circles and dashed line) is also shown here for reference. The vertical dotted line is the distance between neighboring $\mathrm{Mg}$ and $\mathrm{O}$ atoms.

of being range separated, but more general in that the amount of exact exchange approaches a set fraction, instead of zero, at long range [53]. It has been shown to reproduce optical spectra obtained in GW-BSE calculations for semiconductors and insulators [42,54], and its ability to satisfy Koopmans' theorem for gas phase molecules and then reproduce the band gap for molecular solids has been well documented [43,55]. SRSH sets the fraction of long-range exact exchange so that it has the correct $\frac{1}{\epsilon_{\infty} r}$ asymptotic behavior for large $\mathrm{r}$.

For technical reasons the CP2K software package [56] is used for PBE0-TC-LRC calculations and the plane wave Vienna $a b$ initio simulation package (VASP) [57] is used for SRSH calculations. To establish a baseline comparison, we use both codes to calculate defect properties with the HSE functional and a reparametrized HSE functional (fitted to reproduce the band gap of $\mathrm{MgO}$ ). We find that the codes agree to $\sim 0.1 \mathrm{eV}$ for CTL calculations.

\section{B. CP2K calculations}

CP2K uses a primary Gaussian basis set mixed with an auxiliary plane-wave basis set [58]. Double- $\zeta$ Gaussian basis sets [59] were used for all atomic species, alongside Goedecker-Teter-Hutter (GTH) pseudopotentials [60]. To check the convergence of the basis set, a bulk cell of $\mathrm{MgO}$ was calculated with the GTH triple- $\zeta$ basis set, and the band gap was found to agree to within $0.04 \mathrm{eV}$. The $\mathrm{V}$ center $(0 /-1)$ CTL was also calculated with the triple- $\zeta$ basis set and was found to agree with the double- $\zeta$ basis set result to within $0.06 \mathrm{eV}$.

The auxiliary plane-wave cutoff was set to $8843 \mathrm{eV}$ (650 Ry). In CP2K, the auxiliary plane-wave cutoffs set the coarseness of the multigrid used for representing certain quantities. These are converged so as to ensure that energy is calculated reliably within the auxiliary plane-wave representation, and also to ensure an efficient mapping of Gaussian functions 
to grids of different coarseness. The plane-wave cutoff is converged until single-point energy calculations change by less than $0.01 \mathrm{eV}$. The basis-set error (in $\mathrm{CP} 2 \mathrm{~K}$ ) therefore is mostly due to the Gaussian, rather than plane-wave, basis set.

The auxiliary density matrix method (ADMM) [61] is employed to increase the efficiency of nonlocal exchange calculation. The ADMM approximation replaces the Gaussian basis set with a smaller set of faster decaying basis functions (the auxiliary basis set) to speed up evaluation of the electron repulsion integrals (ERIs). The auxiliary density matrix $\hat{P}$ is constructed from the primary density matrix $P$. In general, there is an error introduced by the substitution of the correct density matrix for a smaller auxiliary matrix. The ADMM approximation accounts for this error by assuming it can be accurately captured using a GGA level of approximation. Stated mathematically,

$$
E^{\mathrm{HFX}}[P] \approx E^{\mathrm{HFX}}[\hat{P}]+\left(E^{\mathrm{GGA}}[P]-E^{\mathrm{GGA}}[\hat{P}]\right) .
$$

A full description of the implementation of the PBE0-TCLRC functional can be found in [49], here we give a brief overview of the functional. In a periodic system, with a given k-point mesh, the Hartree-Fock exchange is calculated as

$$
\begin{aligned}
E_{\mathrm{X}}= & -\frac{1}{2 N_{\mathbf{k}}} \sum_{i, j} \sum_{\mathbf{k}, \mathbf{k}^{\prime}} \iint \phi_{i}^{\mathbf{k}}(r) \phi_{j}^{\mathbf{k}^{\prime}}(r) g\left(\left|r-r^{\prime}\right|\right) \\
& \times \phi_{i}^{\mathbf{k}}\left(r^{\prime}\right) \phi_{j}^{\mathbf{k}^{\prime}}\left(r^{\prime}\right) d^{3} r d^{3} r^{\prime},
\end{aligned}
$$

with, conventionally, $g\left(\left|r-r^{\prime}\right|\right)=\frac{1}{\left|r-r^{\prime}\right|}$. In a $\Gamma$-point calculation, Eq. (2) reduces to

$$
\begin{aligned}
E_{\mathrm{X}}^{\Gamma}= & -\frac{1}{2} \sum_{i, j} \iint \phi_{i}^{0}(r) \phi_{j}^{0}(r) g\left(\left|r-r^{\prime}\right|\right) \\
& \times \phi_{i}^{0}\left(r^{\prime}\right) \phi_{j}^{0}\left(r^{\prime}\right) d^{3} r d^{3} r^{\prime} .
\end{aligned}
$$

In the PBE0-TC-LRC functional, the potential $g\left(\left|r-r^{\prime}\right|\right)$ is replaced by the truncated Coulomb operator,

$$
g_{\mathrm{TC}}\left(\left|r-r^{\prime}\right|\right)= \begin{cases}\frac{1}{\left|r-r^{\prime}\right|}, & \left|r-r^{\prime}\right| \leqslant R_{c}, \\ 0, & \left|r-r^{\prime}\right|>R_{c} .\end{cases}
$$

Beyond the cutoff radius $R_{c}$, a "long-range correction" (LRC) is applied to replace the lost long-range exchange interaction. This correction is calculated using the long-range part of a semilocal density functional. Specifically, the spherically averaged PBE exchange hole $\rho_{\mathrm{x}}^{\mathrm{SA}}(\mathbf{r}, u)$ is used. The longrange correction to the exchange energy is then

$$
E_{\mathrm{X}}^{\mathrm{PBE}-\mathrm{LRC}}[\rho]=\frac{1}{2} \int \rho(\mathbf{r}) d \mathbf{r} \int_{R_{C}}^{\infty} 4 \pi u \rho_{\mathrm{x}}^{\mathrm{SA}}(\mathbf{r}, u) d u .
$$

where $u$ is the electron-electron distance. This then allows us to write the full expression for the exchange-correlation energy as

$$
\begin{aligned}
E_{\mathrm{XC}}^{\mathrm{PBE} 0-\mathrm{TC}-\mathrm{LRC}}= & \alpha E_{\mathrm{X}}^{\mathrm{HF}-\mathrm{TC}}+\alpha E_{\mathrm{X}}^{\mathrm{PBE}-\mathrm{LRC}} \\
& +(1-\alpha) E_{\mathrm{X}}^{\mathrm{PBE}}+E_{\mathrm{C}}^{\mathrm{PBE}},
\end{aligned}
$$

where $E_{\mathrm{X}}^{\mathrm{HF}-\mathrm{TC}}$ is given by Eq. (3) with $g\left(\left|r-r^{\prime}\right|\right)$ substituted by the truncated Coulomb interaction $g_{\mathrm{TC}}$, and $\alpha$ is the fraction of exact exchange.

\section{VASP calculations}

VASP calculations use PBE-based projector-augmented waves (PAWs) for treating core electrons [62]. The lithium PAW includes the $1 s$ states as valence. The plane-wave cutoff used is $600 \mathrm{eV}$ and the $k$-grid/supercell size (in the case of a gamma-point calculation) is $4 \times 4 \times 4$. The electronic selfconsistency threshold is set to $10^{-5} \mathrm{eV}$ and the number of FFT grid points along one axis is set to twice the number of plane waves. With these parameters, band gaps are converged to $0.02 \mathrm{eV}$, the ion-clamped dielectric constant $\epsilon_{\infty}$ is converged to \pm 0.002 , and the dielectric constant including ionic screening $\epsilon_{0}$ is converged to \pm 0.02 [63], VBM and defect eigenvalues, as well as total energy differences between defect centers of different charges, are converged to about $0.003 \mathrm{eV}$ (with respect to the plane-wave cutoff), and forces are converged to about $10^{-5} \mathrm{eV} / \AA$. When performing ionic relaxations, forces are relaxed to $0.02 \mathrm{eV} / \AA$.

The SRSH functional [43] partitions the exchange part of the Coulomb interaction using the identity [64]

$$
\begin{aligned}
\frac{1}{r}= & \underbrace{\alpha \frac{\operatorname{erfc}(\gamma r)}{r}}_{\mathrm{xx}}+\underbrace{(1-\alpha) \frac{\operatorname{erfc}(\gamma r)}{r}}_{\mathrm{KSx}} \\
& +\underbrace{\frac{1}{\epsilon_{\infty}} \frac{\operatorname{erf}(\gamma r)}{r}}_{\mathrm{xx}}+\underbrace{\left(1-\frac{1}{\epsilon_{\infty}}\right) \frac{\operatorname{erf}(\gamma r)}{r}}_{\mathrm{KSx}},
\end{aligned}
$$

with exchange owing to the first and third terms treated by the exact exchange operator ( $\mathrm{xx}$ ) and exchange owing to the second and fourth terms treated by semilocal exchange $(\mathrm{KSx})$, in our case based on the PBE functional. Here $\alpha$ is the parameter controlling how much short-range exact exchange to use and is set to 0.25 , as in the PBE0 and HSE functionals; $\epsilon_{\infty}$ is the high-frequency dielectric constant and controls how much long-range exact exchange to use; and $\gamma$ is the range-separation parameter that determines the transition of the amount of exact exchange from $\alpha$ to $\frac{1}{\epsilon_{\infty}}$ with increasing $r$.

The full form of the SRSH exchange-correlation functional is then

$$
\begin{aligned}
E_{\mathrm{xc}}^{\mathrm{SRSH}}= & \alpha E_{\mathrm{xx}}^{\mathrm{SR}}+(1-\alpha) E_{\mathrm{KSx}}^{\mathrm{SR}} \\
& +\frac{1}{\epsilon_{\infty}} E_{\mathrm{xx}}^{\mathrm{LR}}+\left(1-\frac{1}{\epsilon_{\infty}}\right) E_{\mathrm{KSx}}^{\mathrm{LR}}+E_{\mathrm{KSc}},
\end{aligned}
$$

where the superscripts denote short-range (SR) and longrange (LR) components and $\mathrm{KSc}$ denotes (semi)local correlation.

\section{Computational procedures}

First, we fit the HSE, PBE0-TC-LRC, and SRSH functionals to the experimental band gap of $\mathrm{MgO}, 7.8 \mathrm{eV}$ [65-69], using a conventional unit cell of $\mathrm{MgO}$ with the experimental lattice constant, $4.21 \AA$. The rationale for fitting all functionals based on the same lattice parameter is that this removes the indirect effect of the lattice parameter on each functional. This facilitates the comparison of the degree to which different functionals satisfy Koopmans' theorem. Calculations of the 
TABLE I. Parameters set for each functional tested. Fitted HSE, PBE0-TC-LRC, and SRSH have all been parametrized to reproduce the experimental band gap of $\mathrm{MgO}$ at the experimental lattice parameter. $\alpha$ is the short-range fraction of exact exchange used. $\gamma$ is the range-separation parameter. $1 / \epsilon_{\infty}$ is the fraction of long-range exact exchange used. $R_{c}$ is the truncation radius used to cut off exact exchange. $a_{\text {lat }}$ is the relaxed lattice parameter for each functional (except for HSE which used the experimental lattice parameter). $E_{g}$ is the fundamental band gap when using the lattice parameter $a_{\text {lat }} \epsilon_{\infty}$ is the computed ion-clamped dielectric constant which is used in the image charge correction when calculating deviation from Koopmans' theorem.

\begin{tabular}{|c|c|c|c|c|}
\hline & Functional parameters & $a_{\text {lat }}(\AA)$ & $E_{g}(\mathrm{eV})$ & $\epsilon_{\infty}$ \\
\hline HSE (CP2K/VASP) & $\alpha=0.25, \gamma=0.2 \AA^{-1}$ & $4.23 / 4.21$ & $6.34 / 6.48$ & $2.52 / 2.91$ \\
\hline Fitted HSE (CP2K) & $\alpha=0.445, \gamma=0.2 \AA^{-1}$ & 4.20 & 7.81 & 2.39 \\
\hline Fitted HSE (VASP) & $\alpha=0.432, \gamma=0.2 \AA^{-1}$ & 4.17 & 8.16 & 2.77 \\
\hline PBE0-TC-LRC & $\alpha=0.325, R_{c}=6.0 \AA$ & 4.21 & 7.77 & 2.47 \\
\hline SRSH & $\alpha=0.25, \frac{1}{\epsilon_{\infty}}=0.33, \gamma=0.75 \AA^{-1}$ & 4.19 & 7.94 & 2.87 \\
\hline
\end{tabular}

zero-point renormalization of the band gap for $\mathrm{MgO}$ yield values of $0.22-0.53 \mathrm{eV}$ [70-72]. In light of this range, and the fact that it is not clear how to include zero-point renormalization effects into a CTL calculation self-consistently, we choose not to include the zero-point renormalization in the value of the experimental band gap for the fitting procedure. For the fitted HSE functional, the fraction of exact exchange in the short range is increased until the correct band gap is obtained. For PBE0-TC-LRC, the fraction of exact exchange is set to be 0.325 , which has been used in previous studies [51,73] and, given the experimental high-frequency dielectric constant of 3.0 [74-76], is also close to $\frac{1}{\epsilon_{\infty}}$. The cutoff radius for the exact exchange is increased until the correct band gap is obtained. For SRSH, the long-range fraction of exact exchange is set based on the experimental high-frequency dielectric constant to be 0.33 [77]. The range-separation parameter $\gamma$ is fit so that the band gap is reproduced. A summary of the parameters used for each functional is given in Table I.

Second, we relax the lattice parameter for the fitted HSE, SRSH, and PBE0-TC-LRC functionals. For HSE we use the experimental lattice parameters to avoid additional errors that would complicate comparisons between the two software packages. The relaxed lattice parameters and the band gaps using the relaxed lattice parameters are also listed in Table I.

Third, we calculate $\epsilon_{\infty}$ and $\epsilon_{0}$ for use in image charge corrections of charged supercells. The reason we use calculated dielectric constants rather than the experimental values is that this causes calculations checking the satisfaction of Koopmans' theorem (also called Koopmans' compliance) for small finite supercells to approach the limit of an infinite supercell more rapidly. In both VASP and $\mathrm{CP} 2 \mathrm{~K}, \epsilon_{\infty}$ is calculated by the change in polarization in response to a small electric field using a gamma point calculation for a cell of fixed geometry (see Table I). $\epsilon_{0}$ is calculated in VASP using the same approach, but with the ions allowed to move in response to the electric field.

Fourth, we create $4 \times 4 \times 4$ cubic supercells, which contain 512 atoms, with an [Li], V, or F (an oxygen vacancy) center defect and relax the geometry (all calculations are done at the $\Gamma$ point). $[\mathrm{Li}]^{0}$ and $\mathrm{V}^{-1}$ centers have a hole trapped on one of the six oxygen ions surrounding the defect. This causes distortions of the order of $0.1 \AA$ for nearby ions, as well as long-range distortions several unit cells away. For this reason, CTL calculations require large supercells to accurately calculate total energy differences. In order to relax the ionic positions to the global minimum, we start with an initial guess in which we move neighboring $\mathrm{Mg}$ ions $0.1 \AA$ away from the hole-localizing oxygen ion. The initial guess is created using the atomic simulation environment (ASE) package [78].

Finally, CTLs and Koopmans' compliance errors are calculated for the defects as described below. $\mathrm{CP} 2 \mathrm{~K}$ is used to check the size of the supercell needed to converge CTL energies. It is found that CTL energies calculated using Eq. (9) for the $5 \times 5 \times 5$ supercell (1000 atoms) differ very little from those computed with the $4 \times 4 \times 4$ (512 atoms) supercell, with the CTL energies increasing by no more than $0.05 \mathrm{eV}$ for the larger supercells. The image charge correction applied to these calculations varies slowly with supercell size and differs by no more than $0.03 \mathrm{eV}$ between the $5 \times 5 \times 5$ and the $4 \times 4 \times 4$ supercells.

Additionally, we quantify the amount of the hole polaron charge residing on a single oxygen atom using Bader charge analysis [79]. This analysis, while physically intuitive, is still just one way to characterize the degree of hole localization and we attach more importance to the change of localization from functional to functional rather than to absolute values. When calculating Bader charges using VASP, we include the charge density associated with the core electrons and make sure that the Bader charge is converged to 0.01 electrons with respect to the FFT grid used to evaluate the charge density.

\section{E. Charge transition levels}

Charge transition levels (CTLs) are formally defined as the Fermi energy level at which the formation energies of a defect center $D$ for two charge states $q_{1}$ and $q_{2}$ are equal [1-3]. This means that if the Fermi energy $\epsilon_{F}$ is lower than the CTL [denoted as $\epsilon_{D}\left(q_{1} / q_{2}\right)$ ], then the defect is in the state $D^{q_{1}}$. If the Fermi energy is higher than $\epsilon_{D}\left(q_{1} / q_{2}\right)$, then the defect is in the state $D^{q_{2}}$. CTLs are calculated via the following equation $[1-3,80]$ :

$$
\begin{aligned}
\epsilon_{D}\left(q_{1} / q_{2}\right)= & \frac{\left(E\left[D^{q_{2}}\right]+E_{\mathrm{corr}}\left(q_{2}\right)\right)-\left(E\left[D^{q_{1}}\right]+E_{\mathrm{corr}}\left(q_{1}\right)\right)}{q_{1}-q_{2}} \\
& -\left[\epsilon_{\mathrm{VBM}}\left[H^{0}\right]+\Delta V_{D H}\right],
\end{aligned}
$$


where $E\left[D^{q_{1}}\right]$ is the total energy of the defect with charge state $q_{1}\left(q_{1}>0\right.$ means that the defect state is positively charged), $E_{\text {corr }}$ is an image charge correction (see Sec. IIF), $\epsilon_{\mathrm{VBM}}\left[H^{0}\right]$ refers to the valence band maximum of the neutral host system, and $\Delta V_{D H}$ is the difference in the electrostatic potential between the neutral defect system and the neutral host system [81]. The convention used here defines the CTL with respect to the valence band. Thus $\epsilon_{D}\left(q_{1} / q_{2}\right)$ is the energy it takes to move an electron from the valence band into the unoccupied defect level [1]. It should be noted that the ionic positions are relaxed when calculating each $E\left[D^{q}\right]$ in Eq. (9). We calculate $V_{D H}$ via the electrostatic potential of the core of an atom far away from the defect [82].

Koopmans' compliance is trivially obeyed for delocalized orbitals in (semi)local functionals [25,83] and one could equally use $\epsilon_{\mathrm{VBM}}\left[H^{0}\right]$ or $E\left[H^{0}\right]-E\left[H^{1}\right]$ in Eq. (9) [80], where $E\left[H^{1}\right]$ is the total energy of the bulk host with one electron removed [84]. We see that while this is also true for hybrid functionals (see Fig. 1 of the Supplemental Material (SM) [85]), they approach the bulk limit more slowly than semilocal functionals. The SRSH functional has a significant deviation of $-0.3 \mathrm{eV}$ from Koopmans' compliance for a $4 \times 4 \times 4$ supercell. We find that the eigenvalue of the VBM converges more rapidly with respect to the size of the supercell, thus we choose to use the VBM eigenvalue instead of a $\Delta$-SCF calculation in Eq. (9).

\section{F. Image charge correction}

For a total energy calculation of a system with a net charge that uses periodic boundary conditions, a compensating jellium background is added to the calculation to prevent the Hartree term from diverging. This spuriously lowers the total energy of charged systems and a correction term $E_{\text {corr }}$ must be included $[86,87]$. Various correction schemes exist $[82,88,89]$ and we choose the Lany-Zunger correction $[1,80]$ for CTL calculations:

$$
E_{\mathrm{corr}}(q)=(1+f) \frac{\alpha q^{2}}{2 \epsilon L},
$$

where $\alpha=2.8373$ is the Madelung constant for a simple cubic periodic array of point charges in jellium [86], $L$ is the length of one side of the supercell, and $\epsilon$ is the dielectric screening constant. The factor $f$ takes into account the second radial moment of the defect charge distribution. When $f=0$ the LanyZunger correction reduces to a monopole correction. Due to screening in bulk systems, it was observed that the dipole term could be taken into account by setting $f=-0.33[1,80,82]$.

If the total energy being corrected has relaxed ionic positions, we use the static dielectric constant $\epsilon_{0}$. Calculating $\epsilon_{0}$ is expensive, so we use the $\epsilon_{0}$ calculated by SRSH for all VASP calculations. A $10 \%$ variation in $\epsilon_{0}$ would cause less than a $0.03 \mathrm{eV}$ change in the image charge correction. Additionally the value of $\epsilon_{0}$ calculated by SRSH (8.57) is close to the bulk experimental value (9.83) [90]. When correcting calculations which do not relax the ionic positions, we use the highfrequency dielectric constant $\epsilon_{\infty}$. However, it has recently been pointed out that there may be errors associated with this procedure [91].

\section{G. Checking Koopmans' compliance}

Deviation from satisfying Koopmans' theorem [also called Koopmans' compliance (KC)] for a given defect center $D$, as it changes from charge state $q$ to $q+1$, is calculated as follows. We compare the $\Delta$-SCF vertical (fixed-ion) ionization energy for a defect with the eigenvalue that is being depopulated, which is associated with the localized charge on the defect. This comparison takes the form

$$
\begin{aligned}
\Delta K\left[D^{q}\right]= & \left(E\left[D^{q+1}\right]+E_{\mathrm{corr}}(q+1)\right)-\left(E\left[D^{q}\right]+E_{\mathrm{corr}}(q)\right) \\
& +\left(\epsilon_{\mathrm{ho}}\left[D^{q}\right]-\frac{2}{q} E_{\mathrm{corr}}(q)\right),
\end{aligned}
$$

where image charge corrections $E_{\text {corr }}$ have been added where needed [92] and $\epsilon_{\mathrm{ho}}\left[D^{q}\right]$ is the highest occupied orbital for the defect with charge state $q$. When using the eigenvalue from a charged supercell we apply the image charge correction as derived in Ref. [27], as can be seen in the last term on the right-hand side of Eq. (11). It should be noted that both the $D^{q}$ and $D^{q+1}$ calculations in Eq. (11) use the same ionic positions, chosen to ensure that the orbital of the state being depopulated is localized for both calculations.

For the $[\mathrm{Li}]$ center, we calculate $\Delta K\left[[\mathrm{Li}]^{-1}\right]$ using the relaxed ionic positions of the $[\mathrm{Li}]^{0}$ state. Unfortunately, we cannot calculate $\Delta K\left[\mathrm{~V}^{-2}\right]$ because of difficulties associated with the ionic positions. The relaxed ionic positions of the $\mathrm{V}^{-2}$ state do not break the symmetry of the lattice; if we use this configuration to calculate $\Delta K\left[\mathrm{~V}^{-2}\right]$, the calculation of $E\left[\mathrm{~V}^{-1}\right]$ does not converge because the hole cannot be localized on a single site due to symmetry. The relaxed ionic positions of the $\mathrm{V}^{-1}$ state ionically screen the excess -1 charge; if we use this configuration to calculate $\Delta K\left[\mathrm{~V}^{-2}\right]$, it is unclear what image charge correction to use for $E\left[\mathrm{~V}^{-2}\right]$ as the excess charge is only partially ionically screened. To remove doubt, we calculate $\Delta K\left[\mathrm{~V}^{-1}\right]$ using the $\mathrm{V}$ center with bulk ionic positions so that we can use $\epsilon_{\infty}$ in $E_{\text {corr }}$. In addition to [Li] and $\mathrm{V}$ centers, we calculate $\Delta K$ for the oxygen vacancy defect, called an $\mathrm{F}$ center, in order to check if $\Delta K$ for a given functional is the same for several different defects. For the $\mathrm{F}$ center, we calculate $\Delta K\left[\mathrm{~F}^{0}\right]$ using the relaxed ionic positions of the $\mathrm{F}^{0}$ state.

For calculating $\Delta K$, we use the Makov-Payne monopole correction, i.e., $f=0$ in Eq. (10) $[86,87]$. This was found previously to remove the supercell size dependence of the $\mathrm{KC}$ calculations more rapidly [81]. We confirm this finding in Fig. 2 by calculating $\Delta K\left[\mathrm{~F}^{0}\right]$ and $\Delta K\left[[\mathrm{Li}]^{-1}\right]$ for unrelaxed supercells of increasing size. As can be seen in the figure, $\mathrm{KC}$ calculations are very sensitive to the type of image charge correction used, which for our calculations is of the same order of magnitude as the deviation from $\mathrm{KC}$.

Other methods of calculating $\mathrm{KC}$ are discussed in the Supplemental Material (SM). All methods agree to within $0.1 \mathrm{eV}$, which provides confirmation of the validity of the calculations. In addition, band decomposed charge densities of the wave functions of the polarons are used to confirm visually that the highest occupied wave function for charge state $q$ is similar to the difference in the charge density between the two charge states [93]. We note that deviation from $\mathrm{KC}$ is not strongly sensitive to the atomic positions or 


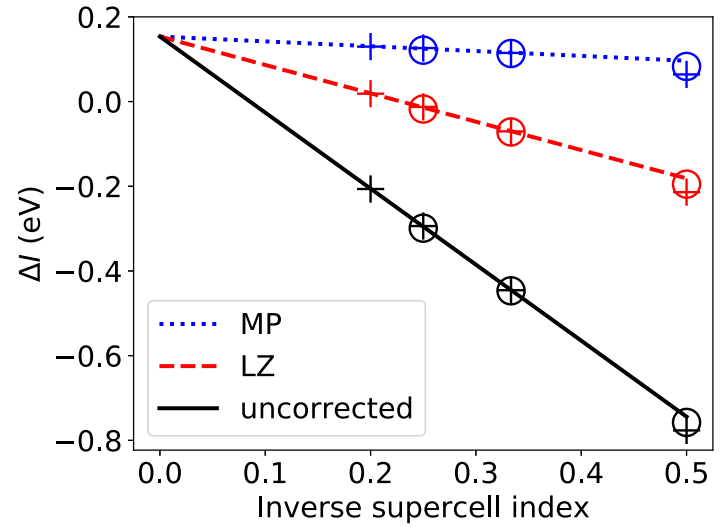

FIG. 2. Deviation from Koopmans' compliance for unrelaxed Li centers (open circles) and F centers (pluses) with respect to the inverse supercell size using the SRSH functional. An $n \times n \times n$ supercell has an inverse supercell index of $1 / n$. Lines are linear fits of the $\mathrm{F}$ center data. Black solid line: Data without image charge corrections. Red dashed line: Data with Lany-Zunger charge corrections. Blue dotted line: Data with Makov-Payne monopole corrections.

lattice parameter. A $1 \%$ change in the lattice parameter of the $\mathrm{F}$ center leads to a $3 \%$ change in $\mathrm{KC}$.

\section{RESULTS AND DISCUSSION}

\section{A. Calculated CTLs compared to experiment}

CTLs for [Li] and V centers calculated using each functional, as well as experimental CTL values, are given in Table II. Even for these well-studied defects, some discrepancies exist in the literature. Therefore, a detailed review of available experimental results is given in the Supplemental Material [85]. In addition, we show the $\Delta-\mathrm{SCF}$ values between the different charge states [the first term on the righthand side of Eq. (9)] and the VBM eigenvalue of the host system in order to identify the cause of differences in the CTL between functionals.

First, we note that HSE CTL results obtained using the two codes in Table II differ by $\pm 0.1 \mathrm{eV}$. This is likely a consequence of the difference between the VASP PAWs and the pseudopotentials used in $\mathrm{CP} 2 \mathrm{~K}$ and the level of numer- ical convergence. The difference between the CTL values calculated by the two codes increases slightly for the fitted HSE functionals because they are independently fitted and the lattice parameter relaxes to a somewhat different value. While CTL values calculated by VASP and CP2K can be compared directly, $\Delta$-SCF and $\epsilon_{\mathrm{VBM}}$ values cannot be compared directly because in each code they are shifted by an arbitrary constant to maintain an average electrostatic potential of zero. These shifts are different mainly because of the use of different pseudopotentials in the two codes.

Second, we see that all the fitted functionals predict larger CTLs than the experimental values, but that the CTLs of SRSH are somewhat closer to experiment than those attained by using fitted HSE or PBE0-TC-LRC, even taking into account a 0.1 to $0.2 \mathrm{eV}$ difference between the codes. This trend is even clearer when comparing only VASP results, i.e., SRSH with fitted HSE. Additionally, we see that the enhanced accuracy of CTLs produced by SRSH, compared to the VASP HSE results, is essentially due to the position of the VBM eigenvalue.

We can rule out trivial explanations for the difference in CTL values. First, each functional uses a relaxed lattice parameter that is slightly different and this affects the band gap (see Table I) and the VBM eigenvalue. However, we also calculated CTLs using the experimental lattice parameter. We found that the CTL values did not change when using the experimental lattice parameter and that the VBM eigenvalues were still responsible for the differences between CTLs. Second, differences in pseudopotentials can affect computed values, but they cannot explain our findings because our conclusions hold independently for the VASP and CP2K data.

In light of this, the difference in the calculated VBM eigenvalues is directly related to the functional used. Despite having very different forms of short-range exact exchange, fitted HSE and PBE0-TC-LRC produce very similar CTLs, $\Delta$ $\mathrm{SCF}$ values, and VBM eigenvalues (using CP2K). Therefore, it is reasonable to conclude that the inclusion of screened long-range exchange is the distinguishing feature between SRSH and the other two fitted functionals. In the next section we offer a plausibility argument for why SRSH's improved CTL is caused by an improved VBM eigenvalue due to including long-range exact exchange, rather than by a simple cancellation of errors.

TABLE II. Charge transition levels (CTLs) for [Li] and V centers in $\mathrm{MgO}$ using different functionals. The CTL calculation is composed of a $\triangle$-SCF term [the first term on the right-hand side of Eq. (9)] minus the VBM eigenvalue (we neglect here the potential alignment term which is of the order of $0.02 \mathrm{eV}$ ). Values are given in eV. Experimental results are taken from Refs. [9,94-96] for [Li] centers and Refs. [7,97,98] for $\mathrm{V}^{-1}$ centers, see the Supplemental Material for more details.

\begin{tabular}{|c|c|c|c|c|c|}
\hline & $\epsilon_{[\mathrm{Li}]}(0 /-1)$ & $\epsilon_{\mathrm{V}}(-1 /-2)$ & $\Delta-\mathrm{SCF}(\mathrm{Li})$ & $\Delta$-SCF (V) & $\epsilon_{\mathrm{VBM}}[H]$ \\
\hline HSE (VASP) & 0.34 & 0.98 & 2.27 & 2.87 & 1.94 \\
\hline SRSH & 0.96 & 1.63 & 2.24 & 2.89 & 1.29 \\
\hline Fitted HSE (VASP) & 1.07 & 1.82 & 2.27 & 2.99 & 1.21 \\
\hline $\mathrm{HSE}(\mathrm{CP} 2 \mathrm{~K})$ & 0.40 & 0.85 & 3.58 & 4.02 & 3.18 \\
\hline PBE0-TC-LRC & 1.21 & 1.88 & 3.57 & 4.24 & 2.36 \\
\hline Fitted HSE (CP2K) & 1.23 & 1.92 & 3.55 & 4.25 & 2.34 \\
\hline Expt. & 0.7 & $1.3-1.6$ & & & \\
\hline
\end{tabular}




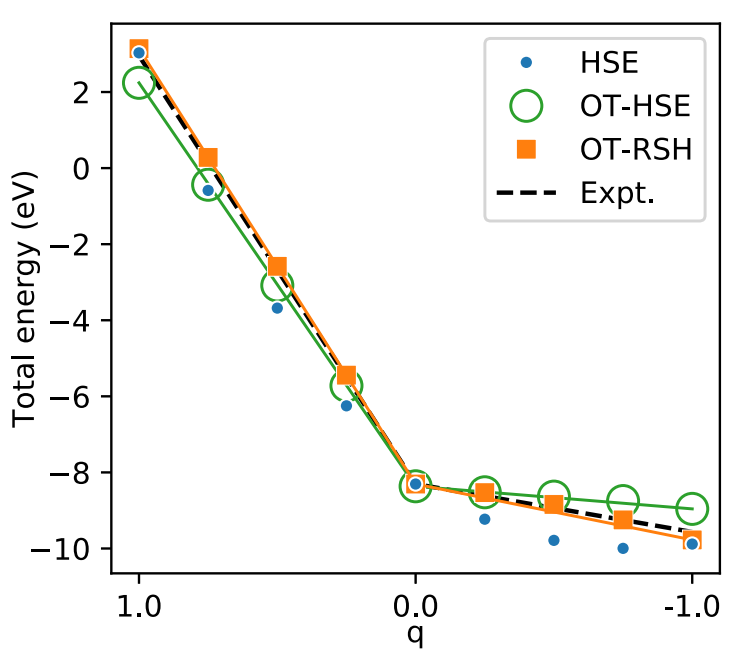

FIG. 3. Total energy (including image charge corrections) of a carbon atom in vacuum as a function of the fractional charge of the atom using different functionals. The lines connect the total energies at $q=1$ to $q=0$, and $q=0$ to $q=-1$. Thus the slope of the line segment from $(1,0)$ is minus the ionization potential, as calculated by $\Delta-\mathrm{SCF}$, and the slope of the line segment from $(0,-1)$ is minus the electron affinity. Deviation from Koopmans' compliance is visually demonstrated by the deviation of the total energy from the straight lines for fractional charges, i.e., curvature. HSE displays significant curvature, whereas optimally tuned HSE (OT-HSE), i.e., HSE adjusted to be Koopmans' compliant, is piecewise linear, albeit at the cost of yielding the wrong ionization potential and electron affinity. The optimally tuned range-separated hybrid functional (OT$\mathrm{RSH}$ ), owing to inclusion of long-range exact exchange, is both Koopmans' compliant and predicts the correct ionization potential and electron affinity. Here the HSE and the experimental data [99] were shifted so that the total energy at $q=0$ equals that of OT-RSH, to facilitate the comparison. Calculations were performed using VASP.

\section{B. Relation of the highest occupied eigenvalue to Koopmans' compliance and long-range exact exchange: An analogy to an atomic system}

To understand the effect of long-range exact exchange on the eigenvalue of the highest occupied orbital, it is instructive to consider an isolated atom and analyze it in terms of KC. There are several reasons why this is illuminating: (i) atoms typically feature large deviations from Koopmans' theorem [23]; (ii) the image charge correction, with $f=0$ and $\epsilon=1$ in Eq. (10), becomes very accurate [86]; (iii) hybrid functionals can be tuned to satisfy Koopmans' theorem unequivocally for these systems [24,41]; and (iv) their analysis is not complicated by dielectric screening, ionic relaxation, and comparing an eigenvalue of one system (the bulk) with total energy differences of another system (a supercell with a defect). Here we consider the lowest triplet state of carbon atom

In Fig. 3 we show total energy as a function of a fractional number of electrons for HSE, optimally tuned HSE (OT-HSE), and the optimally tuned range-separated hybrid functional (OT-RSH) for a carbon atom. The term "optimally tuned" denotes nonempirical adjustment of the functional's parameters so as to satisfy $\mathrm{KC}$, as opposed to all other reparametrized functionals explored in this article, which were fit to reproduce the band gap. More specifically, optimal tuning sets the functional's parameters to satisfy $\Delta K\left[C^{0}\right]=0$ in Eq. (11) (see the SM for more details). We also note that OT-RSH includes full long-range exact exchange so as to satisfy the correct asymptotic $1 / r$ behavior in vacuum. It is apparent in Fig. 3 that the total energy using the HSE functional has significant curvature, but that the $\Delta$-SCF ionization energy and electron affinity, as indicated by the endpoints, are very close to experiment. Next, we see that both OT-HSE and OT-RSH are piecewise linear on the line segment $q \in(1,0)$, confirming that they satisfy $\Delta K\left[C^{0}\right]=0$. OT-HSE corrects the eigenvalue of the highest occupied orbital by greatly increasing short-range exact exchange, and this causes the ionization energy and electron affinity predicted by OT-HSE to be inaccurate (the endpoints and the slopes do not agree with experiment). This means that the eigenvalue gap between the highest occupied molecular orbital and the lowest unoccupied molecular orbital (HOMO-LUMO gap) of OT-HSE is incorrect, even though Koopmans' theorem is obeyed. On the other hand, OT-RSH corrects the eigenvalue using long-range exact exchange and does not noticeably affect the ionization potential and electron affinity predicted by $\Delta$-SCF $[3,38,39]$, meaning that Koopmans' theorem is obeyed and the HOMOLUMO gap is also correct. Since the real ionization process moves an electron out to infinity, it makes physical sense that enforcing the correct long-range behavior by including the right fraction of long-range exact exchange improves the accuracy of the eigenvalues for the right reason, without negatively affecting the ionization potential and electron affinity calculated by $\Delta$-SCF [24].

Returning to $\mathrm{MgO}$, SRSH yields a VBM eigenvalue which improves the calculated CTLs while yielding essentially the same $\Delta$-SCF values as HSE. In contrast, fitted HSE and PBE0-TC-LRC yield VBM eigenvalues that cause the CTLs to be somewhat overestimated and yield $\Delta$-SCF values for the $\mathrm{V}$ center which differ from those given by HSE, further contributing to the overestimation of the CTLs. This is the same overall behavior as for the carbon atom. This strongly suggests that SRSH yields improved CTLs because including screened long-range exact exchange improves the VBM eigenvalue of the bulk system.

Based on the above considerations, we suggest that for functionals with screened long-range exact exchange, the overall amount of exact exchange that makes the bulk system Koopmans' compliant will also yield the correct band gap. One can then tune either quantity to achieve accurate VBM eigenvalues. We clarify that, as noted earlier, Koopmans' compliance is trivially obtained for the bulk system in the infinite supercell limit. Here a functional being Koopmans' compliant for the bulk means that the exchange-correlation functional is not missing a derivative discontinuity for that parametrization of exact exchange. How to calculate the deviation from Koopmans' compliance for a bulk system is an active area of research $[83,100]$. Lorke et al. [101] reported a related finding, where a hybrid functional fit to have the correct long-range behavior and band gap was also Koopmans' compliant for a defect. Screened long-range exact exchange is most relevant for ionic insulators owing to their relatively small high-frequency dielectric constant. In contrast, the fact 
TABLE III. Deviation from Koopmans' compliance for several defects, in eV; charge transition levels (CTLs), in eV (from Table II for convenience); degree of localization of the polaron, measured by the fraction of the hole charge localized on one oxygen site as calculated by Bader charge analysis.

\begin{tabular}{lcccccr}
\hline \hline & $\Delta K\left[[\mathrm{Li}]^{-1}\right]$ & $\Delta K\left[\mathrm{~V}^{-1}\right]$ & $\Delta K\left[\mathrm{~F}^{0}\right]$ & $\epsilon_{[\mathrm{Li}]}(0 /-1)$ & $\epsilon_{\mathrm{V}}(-1 /-2)$ & ${\text { Loc. }[\mathrm{Li}]^{0}}^{\text {Loc. V }}$ \\
\hline HSE (VASP) & 0.81 & - & 0.69 & 0.34 & 0.98 & 0.53 \\
SRSH & 0.18 & 0.06 & 0.13 & 0.96 & 1.63 & 0.62 \\
Fitted HSE (VASP) & 0.09 & 0.24 & 0.45 & 1.07 & 1.82 & 0.59 \\
HSE (CP2K) & 0.89 & - & 0.64 & 0.40 & 0.85 & 0.68 \\
PBE0-TC-LRC & 0.10 & 0.15 & 0.44 & 1.21 & 1.88 & 0.69 \\
Fitted HSE (CP2K) & 0.25 & 0.24 & 0.61 & 1.23 & 1.92 & 0.72 \\
\hline \hline
\end{tabular}

that HSE yielded accurate band gaps, obeyed Koopmans' theorem, and yielded accurate CTLs for materials with a large high-frequency dielectric constant [30], may indicate that having no exact exchange in the long-range is an acceptable approximation.

\section{Relation between Koopmans' compliance of defect systems and the accuracy of calculated CTLs}

Given that we fit the functionals in this study to the band gap and not to $\mathrm{KC}$, we investigate the relationship between the $\mathrm{KC}$ for the defect system and the accuracy of the CTLs, as summarized in Table III. We see that fitting the functional to yield the correct band gap does indeed reduce the deviation from $\mathrm{KC}$. We also see that functionals with lower $\Delta K$ (i.e., less convex) predict larger CTLs as well as greater localization of the hole on a single oxygen site. More quantitatively, the differences in $\mathrm{KC}$ between the functionals for the [Li] center match the differences between corresponding CTL values as calculated by VASP. The trend is less clear for $\mathrm{KC}$ of the $\mathrm{V}$ centers, especially since HSE was not able to localize the hole at all. This can be attributed to the fact that the $\mathrm{KC}$ calculations are not using the same geometry as the CTLs, and hence are looking at the different character of polaron localization.

While it is clear from $\Delta K$ data for HSE that gross deviation from $\mathrm{KC}$ yields inaccurate CTLs, two observations lead us to suggest that the $\mathrm{KC}$ of the defect system is not directly indicative of the accuracy of the calculated CTL, in particular for functionals that do not have screened long-range exact exchange. First, the deviation from $\mathrm{KC}$ of SRSH for the [Li] defect is about $0.1 \mathrm{eV}$ worse than the $\mathrm{KC}$ of fitted HSE (calculated by VASP), despite the fact that the [Li] CTL calculated by SRSH is closer to experiment. Second, KC varies by $0.3 \mathrm{eV}$ between defects using the fitted HSE functional. The first observation may be simply due to errors in the image charge corrections and by itself does not prove that the $\mathrm{KC}$ of a defect does not directly indicate the accuracy of the CTL value. Image charge corrections are still an area of active research [91] and it is somewhat surprising that Makov-Payne corrections reduce the size dependence of $\Delta K$ calculations better than Lany-Zunger corrections. Thus, it is possible that better corrections exist that can shift our KC calculations.

However, the second observation shows that a more subtle problem also plays a part in explaining why in our case the calculated $\mathrm{KC}$ is somewhat worse for the SRSH functional, despite it having more accurate CTLs. A distinction must be made between the eigenvalue being examined in $\Delta K$ calculations, which belongs to the localized defect, and the VBM eigenvalue of the bulk system, which is used in the CTL calculations. There is only one value of $\alpha$ for the fitted HSE functional that will minimize the derivative discontinuity for the bulk VBM eigenvalue [22]. The variation in $\Delta K$ associated with different defects for the fitted HSE functional indicates that the $\alpha$ that minimizes the derivative discontinuity of the VBM is likely not the same $\alpha$ that reduces $\Delta K\left[[\mathrm{Li}]^{-1}\right]$. Thus the error in a CTL calculation due to the VBM eigenvalue is not directly correlated to satisfaction of $\mathrm{KC}$ for a given defect. We note that others have also observed that different defects require somewhat different fractions of exact exchange to satisfy Koopmans' theorem [29] and that a better image charge correction is unlikely to greatly reduce the variation of $\Delta K$ for different defects. The fact that SRSH has a smaller spread in KC between defects may be a further indication that including screened long-range exact exchange facilitates the possibility of satisfying several conditions simultaneously.

Finally, in Table III we can also see that all the hybrid functionals investigated here localize hole polarons in relaxed [Li] and $\mathrm{V}$ centers in $\mathrm{MgO}$, even $\mathrm{HSE}$, which shows significant convexity $(\Delta K>0)$. There are two hints that SRSH describes polaron localization more accurately. First, functionals with increased short-range exact exchange yield greater localization of the polaron, as reported in Table III. In the case of the $\mathrm{V}$ center we see that the larger amount of short-range exact exchange corresponds to larger $\triangle-S C F$ components and that this contributes to the overestimation of the V center CTL (see Table II). Therefore large amounts of short-range exact exchange may be overlocalizing the hole polaron. Second, the SRSH functional does not localize self-trapped holes in $\mathrm{MgO}$, in agreement with experimental evidence [11,98,102], whereas the PBE0-TC-LRC functional does localize a selftrapped hole, albeit with a small trapping energy of $0.36 \mathrm{eV}$.

\section{Remaining sources of error}

While the SRSH functional yields the most accurate CTLs, it is still $0.25 \mathrm{eV}$ larger than experiment for [Li] centers and possibly also for $\mathrm{V}$ centers, raising the question of what the remaining sources of error are. Pseudopotentials and code implementations can give a $0.2 \mathrm{eV}$ error, as demonstrated by the the CTL energies of the independently fitted HSE functionals calculated by both DFT codes. The supercell size may not be fully converged. This is partly due to the use of approximate image charge corrections. It may also 
be due to errors introduced by elastic effects when performing ionic relaxation, stemming from the finite size of the supercell. CTLs calculated using a larger supercell size $(5 \times 5 \times 5)$ increased by no more than $0.05 \mathrm{eV}$. Based on the SRSH $\Delta K$ values for different defect centers, we estimate that the $\mathrm{KC}$ error of the VBM eigenvalue is of the order of $0.1-0.2 \mathrm{eV}$. Beyond numerics, zero-point renormalization and other phonon-electron coupling effects may need to be explicitly included for improved accuracy and obviously the approximate functional itself introduces an error.

\section{CONCLUSION}

We calculated charge transition levels of several wellstudied hole polaron defects in $\mathrm{MgO}$ with several hybrid functionals. The results provide clear evidence that a functional fitted to the fundamental band gap of the material yields somewhat more accurate CTLs, as compared to experiment, if it includes screened long-range exact exchange. We attribute this improvement mainly to an improved VBM eigenvalue. In addition, we do not find a direct correlation between the accuracy of a CTL and the degree to which Koopmans' theorem is satisfied for that defect for the fitted hybrid functionals.

Based on these results, for low dielectric constant materials, we recommend calculating defect CTLs, and possibly also trapping energies of self-trapped polarons, using range-separated hybrid functionals with screened long-range exact exchange, fit to the bulk band gap. This method of selecting the parameters of a hybrid functional does not depend on image charge corrections, and it is computationally cheaper than fitting the parameters based on Koopmans' compliance of a defect, while achieving similar or possibly even better accuracy. Because the improvement obtained upon using screened long-range exact exchange is not large, we suspect that any hybrid functional which includes a fraction of long-range exact exchange close to $1 / \epsilon_{\infty}$ will produce reasonable results when fit to the band gap. However, further work is needed to assess whether the conclusions drawn from this initial test case generally hold true for wide band gap insulators.

\section{ACKNOWLEDGMENTS}

The authors acknowledge support by a Weizmann-UK grant. L.K. is the incumbent of the Aryeh and Mintzi Katmann Professorial Chair. J.S. and A.L.S. acknowledge funding provided by the UK Engineering and Physical Sciences Research Council (EPSRC) under Grants No. EP/K01739X/1 and No. EP/P013503/1 and by the Leverhulme Trust RPG-2016-135. Computer facilities on the ARCHER UK National Supercomputing Service have been provided via the UKs HPC Materials Chemistry Consortium (EPSRC Grants No. EP/L000202 and No. EP/R029431).
[1] S. Lany and A. Zunger, Phys. Rev. B 78, 235104 (2008), note that Eq. (2) therein should have $+E_{V}$ and not $-E_{V}$.

[2] C. G. Van de Walle and J. Neugebauer, J. Appl. Phys. 95, 3851 (2004).

[3] C. Freysoldt, B. Grabowski, T. Hickel, J. Neugebauer, G. Kresse, A. Janotti, and C. G. Van de Walle, Rev. Mod. Phys. 86, 253 (2014).

[4] A. Alkauskas, M. D. McCluskey, and C. G. Van de Walle, J. Appl. Phys. 119, 181101 (2016).

[5] I. G. Austin and N. F. Mott, Adv. Phys. 50, 757 (2001).

[6] D. Emin, in Polarons (Cambridge University Press, Cambridge, 2013), p. 211.

[7] O. F. Schirmer, J. Phys.: Condens. Matter 18, R667 (2006).

[8] O. F. Schirmer, J. Phys. Condens. Matter 23, 334218 (2011).

[9] M. M. Tardío, R. Ramírez, R. González, and Y. Chen, Phys. Rev. B 66, 134202 (2002).

[10] N. A. Richter, F. Stavale, S. V. Levchenko, N. Nilius, H.-J. Freund, and M. Scheffler, Phys. Rev. B 91, 195305 (2015).

[11] K. A. Kalder, T. N. Kyarner, C. B. Lushchik, A. F. Malysheva, and R. V. Milenina, J. Appl. Spectrosc. 25, 1250 (1976).

[12] A. J. E. Rettie, W. D. Chemelewski, D. Emin, and C. B. Mullins, J. Phys. Chem. Lett. 7, 471 (2016).

[13] G. Pacchioni, F. Frigoli, D. Ricci, and J. A. Weil, Phys. Rev. B 63, 054102 (2000).

[14] J. Lægsgaard and K. Stokbro, Phys. Rev. Lett. 86, 2834 (2001).

[15] J. L. Gavartin, P. V. Sushko, and A. L. Shluger, Phys. Rev. B 67, 035108 (2003).

[16] S. Lany and A. Zunger, Phys. Rev. B 80, 085202 (2009).

[17] W. H. Sio, C. Verdi, S. Poncé, and F. Giustino, Phys. Rev. Lett. 122, 246403 (2019).
[18] J. B. Varley, A. Janotti, C. Franchini, and C. G. Van de Walle, Phys. Rev. B 85, 081109(R) (2012).

[19] J. P. Perdew, R. G. Parr, M. Levy, and J. L. Balduz, Phys. Rev. Lett. 49, 1691 (1982).

[20] J. P. Perdew and M. Levy, Phys. Rev. Lett. 51, 1884 (1983).

[21] L. J. Sham and M. Schlüter, Phys. Rev. Lett. 51, 1888 (1983).

[22] A. Seidl, A. Görling, P. Vogl, J. A. Majewski, and M. Levy, Phys. Rev. B 53, 3764 (1996).

[23] T. Stein, J. Autschbach, N. Govind, L. Kronik, and R. Baer, J. Phys. Chem. Lett. 3, 3740 (2012).

[24] L. Kronik, T. Stein, S. Refaely-Abramson, and R. Baer, J. Chem. Theory Comp. 8, 1515 (2012).

[25] P. Mori-Sánchez, A. J. Cohen, and W. Yang, Phys. Rev. Lett. 100, 146401 (2008).

[26] S. Lany and A. Zunger, Modell. Simul. Mater. Sci. Eng. 17, 084002 (2009).

[27] W. Chen and A. Pasquarello, Phys. Rev. B 88, 115104 (2013).

[28] P. Deák, Q. Duy Ho, F. Seemann, B. Aradi, M. Lorke, and T. Frauenheim, Phys. Rev. B 95, 075208 (2017).

[29] G. Miceli, W. Chen, I. Reshetnyak, and A. Pasquarello, Phys. Rev. B 97, 121112(R) (2018).

[30] P. Deák, B. Aradi, T. Frauenheim, E. Janzén, and A. Gali, Phys. Rev. B 81, 153203 (2010).

[31] A. R. Elmaslmane, M. B. Watkins, and K. P. McKenna, J. Chem. Theory Comput. 14, 3740 (2018).

[32] T. Gake, Y. Kumagai, and F. Oba, Phys. Rev. Materials 3, 044603 (2019).

[33] S. Kokott, S. V. Levchenko, P. Rinke, and M. Scheffler, New J. Phys. 20, 033023 (2018).

[34] J. P. Perdew and M. Levy, Phys. Rev. B 56, 16021 (1997). 
[35] J. Heyd, G. E. Scuseria, and M. Ernzerhof, J. Chem. Phys. 118, 8207 (2003); 124, 219906 (2006).

[36] C. Adamo and V. Barone, J. Chem. Phys. 110, 6158 (1999).

[37] M. Ernzerhof and G. E. Scuseria, J. Chem. Phys. 110, 5029 (1999).

[38] Z. Zheng, D. A. Egger, J.-L. Brédas, L. Kronik, and V. Coropceanu, J. Phys. Chem. Lett. 8, 3277 (2017).

[39] M. E. Casida and D. R. Salahub, J. Chem. Phys. 113, 8918 (2000).

[40] T. B. de Queiroz and S. Kümmel, J. Chem. Phys. 141, 084303 (2014).

[41] T. Stein, H. Eisenberg, L. Kronik, and R. Baer, Phys. Rev. Lett. 105, 266802 (2010).

[42] S. Refaely-Abramson, M. Jain, S. Sharifzadeh, J. B. Neaton, and L. Kronik, Phys. Rev. B 92, 081204(R) (2015).

[43] S. Refaely-Abramson, S. Sharifzadeh, M. Jain, R. Baer, J. B. Neaton, and L. Kronik, Phys. Rev. B 88, 081204(R) (2013).

[44] L. Kronik and J. B. Neaton, Annu. Rev. Phys. Chem. 67, 587 (2016).

[45] L. Kronik and S. Kümmel, Adv. Mater. 30, 1706560 (2018).

[46] A. M. Stoneham, J. Gavartin, A. L. Shluger, A. V. Kimmel, D. M. Ramo, H. M. Rønnow, G. Aeppli, and C. Renner, J. Phys.: Condens. Matter 19, 255208 (2007).

[47] J. T. Devreese and A. S. Alexandrov, Rep. Prog. Phys. 72, 066501 (2009).

[48] Y. Chen and M. Abraham, J. Phys. Chem. Solids 51, 747 (1990).

[49] M. Guidon, J. Hutter, and J. VandeVondele, J. Chem. Theory Comput. 5, 3010 (2009).

[50] O. A. Dicks and A. L. Shluger, J. Phys.: Condens. Matter 29, 314005 (2017).

[51] J. Strand, S. K. Chulkov, M. B. Watkins, and A. L. Shluger, J. Chem. Phys. 150, 044702 (2019).

[52] J. H. Skone, M. Govoni, and G. Galli, Phys. Rev. B 93, 235106 (2016).

[53] HSE can be thought of as a special case of the SRSH functional with $\epsilon_{\infty} \rightarrow \infty$.

[54] D. Wing, J. B. Haber, R. Noff, B. Barker, D. A. Egger, A. Ramasubramaniam, S. G. Louie, J. B. Neaton, and L. Kronik, Phys. Rev. Materials 3, 064603 (2019).

[55] A. K. Manna, S. Refaely-Abramson, A. M. Reilly, A. Tkatchenko, J. B. Neaton, and L. Kronik, J. Chem. Theory Comput. 14, 2919 (2018).

[56] J. VandeVondele, M. Krack, F. Mohamed, M. Parrinello, T. Chassaing, and J. Hutter, Comput. Phys. Commun. 167, 103 (2005).

[57] G. Kresse and J. Furthmüller, Phys. Rev. B 54, 11169 (1996).

[58] G. Lippert, J. Hutter, and M. Parrinello, Mol. Phys. 92, 477 (1997).

[59] J. VandeVondele and J. Hutter, J. Chem. Phys. 127, 114105 (2007).

[60] S. Goedecker, M. Teter, and J. Hutter, Phys. Rev. B 54, 1703 (1996).

[61] M. Guidon, J. Hutter, and J. VandeVondele, J. Chem. Theory Comput. 6, 2348 (2010).

[62] G. Kresse and D. Joubert, Phys. Rev. B 59, 1758 (1999).

[63] For dielectric calculations the self-consistency threshold was raised to $10^{-8} \mathrm{eV}$ and the convergence value reported includes convergence with respect to supercell size.
[64] This is equivalent to the more compact representation in previous papers [42], where the $\mathrm{xx}$ and $\mathrm{KSx}$ terms are combined and $\alpha+\beta=1 / \epsilon$.

[65] R. Whited, C. J. Flaten, and W. Walker, Solid State Commun. 13, 1903 (1973).

[66] D. M. Roessler and W. C. Walker, Phys. Rev. 159, 733 (1967).

[67] N.-P. Wang, M. Rohlfing, P. Krüger, and J. Pollmann, Appl. Phys. A 78, 213 (2004).

[68] A. Schleife, C. Rödl, F. Fuchs, J. Furthmüller, and F. Bechstedt, Phys. Rev. B 80, 035112 (2009).

[69] L. X. Benedict, E. L. Shirley, and R. B. Bohn, Phys. Rev. Lett. 80, 4514 (1998).

[70] J. P. Nery, P. B. Allen, G. Antonius, L. Reining, A. Miglio, and X. Gonze, Phys. Rev. B 97, 115145 (2018).

[71] W. R. L. Lambrecht, C. Bhandari, and M. van Schilfgaarde, Phys. Rev. Materials 1, 043802 (2017).

[72] G. Antonius, S. Poncé, E. Lantagne-Hurtubise, G. Auclair, X. Gonze, and M. Côté, Phys. Rev. B 92, 085137 (2015).

[73] D. Z. Gao, A.-M. El-Sayed, and A. L. Shluger, Nanotechnology 27, 505207 (2016).

[74] I. M. Boswarva, Phys. Rev. B 1, 1698 (1970); O. Madelung, Semiconductors: Data Handbook, 3rd ed. (Springer, Berlin, 2004).

[75] J. C. Phillips, Phys. Rev. Lett. 20, 550 (1968); N. W. Ashcroft and N. D. Mermin, Solid State Physics (Holt, Rinehart and Winston, New York, 1976).

[76] M. Shishkin and G. Kresse, Phys. Rev. B 75, 235102 (2007).

[77] The difference between the long-range behavior set for PBE0TC-LRC and SRSH is due to using previous work. We do not think that changing the long-range fraction by 0.005 will have any meaningful effect.

[78] A. H. Larsen, J. J. Mortensen, J. Blomqvist, I. E. Castelli, R. Christensen, M. Dułak, J. Friis, M. N. Groves, B. Hammer, C. Hargus, E. D. Hermes, P. C. Jennings, P. B. Jensen, J. Kermode, J. R. Kitchin, E. L. Kolsbjerg, J. Kubal, K. Kaasbjerg, S. Lysgaard, J. B. Maronsson, T. Maxson, T. Olsen, L. Pastewka, A. Peterson, C. Rostgaard, J. Schiøtz, O. Schütt, M. Strange, K. S. Thygesen, T. Vegge, L. Vilhelmsen, M. Walter, Z. Zeng, and K. W. Jacobsen, J. Phys.: Condens. Matter 29, 273002 (2017).

[79] W. Tang, E. Sanville, and G. Henkelman, J. Phys.: Condens. Matter 21, 084204 (2009).

[80] C. Persson, Y.-J. Zhao, S. Lany, and A. Zunger, Phys. Rev. B 72, 035211 (2005).

[81] T. R. Durrant, Ph.D. thesis, University College London, 2019.

[82] H.-P. Komsa, T. T. Rantala, and A. Pasquarello, Phys. Rev. B 86, 045112 (2012).

[83] V. Vlček, H. R. Eisenberg, G. Steinle-Neumann, L. Kronik, and R. Baer, J. Chem. Phys. 142, 034107 (2015).

[84] $E\left[H^{1}\right]$ does not need an image correction because the excess charge is delocalized.

[85] See Supplemental Material at http://link.aps.org/supplemental/ 10.1103/PhysRevMaterials.4.083808 for a literature review of the experimental results for hole polaron defect centers in $\mathrm{MgO}$, different methods for calculating Koopmans' compliance, and our investigation into bulk Koopmans' compliance. References [103-117] appear in the SM, but not in the main text.

[86] M. Leslie and N. J. Gillan, J. Phys. C: Solid State Phys. 18, 973 (1985). 
[87] G. Makov and M. C. Payne, Phys. Rev. B 51, 4014 (1995).

[88] C. Freysoldt, J. Neugebauer, and C. G. Van de Walle, Phys. Status Solidi B 248, 1067 (2011).

[89] C. Freysoldt, J. Neugebauer, and C. G. Van de Walle, Phys. Rev. Lett. 102, 016402 (2009).

[90] M. Wintersgill, J. Fontanella, C. Andeen, and D. Schuele, J. Appl. Phys. 50, 8259 (1979).

[91] T. Gake, Y. Kumagai, C. Freysoldt, and F. Oba, Phys. Rev. B 101, 020102(R) (2020).

[92] Since $E_{\text {corr }}$ depends on $q^{2}$, the last image charge correction on the right does not diverge when $q=0$.

[93] D. A. Egger, S. Weissman, S. Refaely-Abramson, S. Sharifzadeh, M. Dauth, R. Baer, S. Kümmel, J. B. Neaton, E. Zojer, and L. Kronik, J. Chem. Theory Comput. 10, 1934 (2014).

[94] R. Ramírez, R. González, R. Pareja, and Y. Chen, Phys. Rev. B 55, 2413 (1997).

[95] M. Puma, A. Lorincz, J. F. Andrews, and J. H. Crawford, J. Appl. Phys. 53, 4546 (1982).

[96] Y. Chen, R. Kernohan, J. Boldu, M. Abraham, D. Eisenberg, and J. Crawford, Solid State Commun. 33, 441 (1980).

[97] A. J. Tench and M. J. Duck, J. Phys. C: Solid State Phys. 6, 1134 (1973).

[98] S. A. Dolgov, T. Kärner, A. Lushchik, A. Maaroos, S. Nakonechnyi, and E. Shablonin, Phys. Solid State 53, 1244 (2011).

[99] CRC Handbook of Chemistry and Physics (internet version), 100th ed., edited by J. R. Rumble (CRC/Taylor \& Francis, Boca Raton, FL, 2019).

[100] T. Bischoff, I. Reshetnyak, and A. Pasquarello, Phys. Rev. B 99, 201114(R) (2019).
[101] M. Lorke, P. Deák, and T. Frauenheim, arXiv:1907.08404.

[102] A. L. Shluger, R. W. Grimes, C. R. A. Catlow, and N. Itoh, J. Phys.: Condens. Matter 3, 8027 (1991).

[103] Y. Chen, H. T. Tohver, J. Narayan, and M. M. Abraham, Phys. Rev. B 16, 5535 (1977).

[104] F. Modine, J. Phys. Chem. Solids 40, 305 (1979).

[105] M. M. Abraham, Y. Chen, L. A. Boatner, and R. W. Reynolds, Phys. Rev. Lett. 37, 849 (1976).

[106] M. M. Abraham, Y. Chen, and W. P. Unruh, Phys. Rev. B 9 , 1842 (1974).

[107] W. P. Unruh, Y. Chen, and M. M. Abraham, Phys. Rev. Lett. 30, 446 (1973).

[108] B. Rose and D. Cowan, Solid State Commun. 15, 775 (1974).

[109] L. E. Halliburton, L. A. Kappers, D. L. Cowan, F. Dravnieks, and J. E. Wertz, Phys. Rev. Lett. 30, 607 (1973).

[110] T. Searle and A. Glass, J. Phys. Chem. Solids 29, 609 (1968).

[111] A. Lushchik, T. Kärner, C. Lushchik, K. Schwartz, F. Savikhin, E. Shablonin, A. Shugai, and E. Vasil'chenko, Nucl. Instrum. Methods B 286, 200 (2012).

[112] E. F. Harris and J. H. Crawford, Jr., Phys. Status Solidi (a) 30 , 223 (1975).

[113] A. Bos, Radiat. Meas. 41, S45 (2006).

[114] A. M. Stoneham, Theory of Defects in Solids: Electronic Structure of Defects in Insulators and Semiconductors (Oxford University Press, Oxford, 2001), Chap. 18.

[115] A. L. Shluger, E. N. Heifets, J. D. Gale, and C. R. A. Catlow, J. Phys.: Condens. Matter 4, 5711 (1992).

[116] J. F. Janak, Phys. Rev. B 18, 7165 (1978).

[117] T. N. Kyarner, A. F. Malysheva, A. A. Maaroos, and V. V. Myurk, Fiz. Tverd. Tela 22, 1178 (1980). 